\title{
As IVF becomes more common, some concerns remain
}

An increasing number of infertile couples have turned to assisted reproduction technology, which facilitates the union of the sperm and the egg. But, in recent years, controversial reports of birth defects in babies conceived through assisted reproduction have led a few researchers to raise concerns about the technology's safety.

Assisted reproduction includes a handful of procedures, many of which are based on in vitro fertilization (IVF) of the egg. In the US alone, assisted reproduction accounted for slightly more than $1 \%$ of all births in 2005, according to the country's Centers for Disease Control and Prevention. The IVF process poses some minor risks to women, including ovarian cysts, mood changes and hot flashes. But a few rare risks to the fetus have given some fertility researchers pause.

Tinkering with sex cells and embryos outside the body, scientists worry, might spur genetic changes that manifest as congenital birth defects. No direct evidence supports that notion, but epidemiological studies have shored up possible links between assisted reproduction and rare genetic syndromes in newborns, such as Beckwith-Wiedemann syndrome, a condition marked by premature birth, an enlarged tongue and heightened susceptibility to tumors, respiratory and speech defects (Hum. Reprod. Update 10, 3-18; 2004).

Fortunately, the syndrome is rare: it normally affects about 1 out of every 12,000 newborns worldwide. But a study found that 3 out 65 US children afflicted with the syndrome had been conceived through IVF (Am. J. Hum. Genet. $72,156-160 ; 2003)$. In other studies, 6 out of 149 children in British and French medical registries of the syndrome were found to have been conceived through IVF or through a

A more experimental option is to remove strips of ovarian tissue, freeze them, and transplant them back into the woman after treatment. Although researchers are still trying to figure out how to reconnect the tissues to the blood supply, at least four women have given birth after an ovarian transplant. This

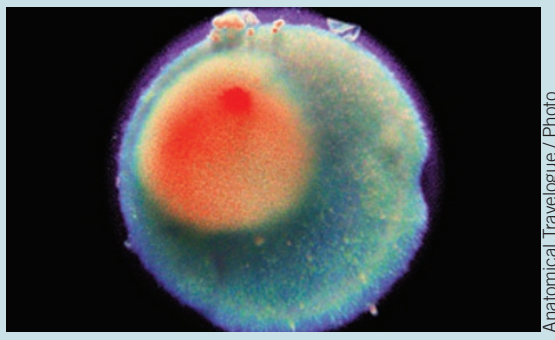

Precious commodity: The human egg specialized technique called intracytoplasmic sperm injection, or ICSI (J. Med. Genet. 40, 62-64; 2003; Am. J. Hum. Genet. 72, 1338-1341; 2003).

"There appeared to be more children with the syndrome conceived through ART [assisted reproductive technology] than we might have expected by chance alone," says Eamonn Maher, a geneticist at the University of Birmingham, UK. Many cases of Beckwith-Wiedemann syndrome, Maher says, stem from abnormalities in DNA methylation — addition of a chemical tag called a methyl group-occurring in specific genes on chromosome 11.

Methylation is one of an array of DNA markers, called imprints, that guide normal development of the embryo. For example, imprinting seems crucial to proper brain growth, says Carmen Williams, a clinical investigator at the US National Institute of Environmental Health Sciences in North Carolina. "The concern is that while the embryo is being cultured in the [IVF] lab, maybe the imprint marks are being changed. We know for sure that happens in mice," she adds (Biol. Reprod. 62, 1526-1535; 2000).

Some studies have suggested a causal relationship between ICSI and abnormal methylation patterns (Am. J. Hum. Genet. 71, 162-164; 2002; Am. J. Hum. Genet. 72, 218-219; 2003). And Maher says the findings hint that imprinting defects might trigger BeckwithWiedemann syndrome.

But he also cautions that the absolute risk of giving birth to a child with BeckwithWiedemann syndrome is low.

"The disease is so rare that it's difficult to counsel an infertile couple not to go forth with ART," Williams says.

last method also holds the greatest promise for children. "You're not going to do embryo freezing with a ten-year-old," notes Oktay.

In the pipeline are also several drugs that promise to protect ovaries from the effects of chemotherapy. In one trial, Kate Stern of the Royal Women's Hospital in Melbourne and her colleagues gave 18 women, with an average age of 25 , a drug called cetrorelix. The drug blocks gonadotropin-releasing hormone, effectively sending ovaries into a prepubertal state. One year after treatment, $94 \%$ of the women were ovulating normally, the researchers reported at a conference in July.

However, because it was a proof-of-concept study, the trial did not include a control group. In their mid-20s, notes Oktay, many women

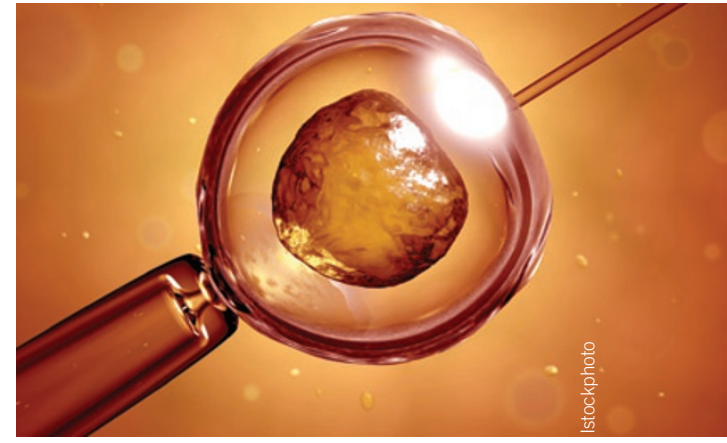

The magic moment: Fertilization of the egg

Conclusive evidence for the possible adverse effects of IVF is unavailable, owing to the dearth of long-term follow up of babies conceived through the technology. One Canadian study reported at the Society for Maternal-Fetal Medicine's conference in 2007 found that babies conceived through IVF were nearly $60 \%$ more likely to develop birth defects than naturally conceived ones. Most of the defects were gastrointestinal, although some were bone, muscle or heart related. Another study, from the University of Iowa, found birth defects in about $6.2 \%$ of about 1,500 IVF-conceived children, in contrast to $4.4 \%$ among naturally conceived ones (Fertil. Steril. 84, 1308-1315; 2005).

If future studies bear out these links, Williams suggests that one can perhaps decrease the risk to the child by avoiding certain invasive procedures that might not be necessary depending on individual circumstances, such as biopsies of implanted embryos, culturing embryos in the lab longer than the minimal time period and using ICSI in the absence of male fertility problems.

Prashant Nair, Chapel Hill, North Carolina do ovulate after chemotherapy, but their egg reserve is diminished, and they go into early menopause. It's too early to say whether this might also happen with the participants in the Australian trial. Stern says her team is set to launch a randomized clinical trial to test cetrorelix further.

Other candidate treatments, such as the compound sphingosine-1-phosphate, prevent the eggs from self-destructing in response to radiation, but research on those candidates has for the most part been limited to animal models. Pending further studies on these compounds, experts say the best option for preserving fertility in women is still to freeze embryos or eggs.

Apoorva Mandavilli, New York 